\title{
El Aprendizaje Basado en Proyectos y su relación con el desarrollo de competencias asociadas al trabajo colaborativo
}

\author{
" Learning based on projects and their relationship \\ with the development of competences associated \\ with collaborative work
}

\begin{tabular}{l}
$\frac{\text { Ernesto Fajardo Pascagaza }}{\text { Universidad Militar - Universidad Santo Tomás }}$ \\
$\frac{\text { BDayron Gil Bohórquez }}{\text { Universidad Francisco José de Caldas }}$ \\
\hline
\end{tabular}

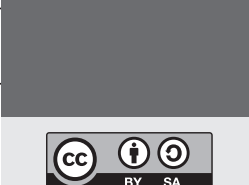

() 2019

\section{Resumen}

A través del tiempo se han propuesto y hoy se siguen creando e implementando numerosos métodos de enseñanza y aprendizaje, buscando que los estudiantes vayan más allá de la memorización de conceptos o teorías y puedan analizar e interpretar el mundo de forma crítica y responsable. El aprendizaje basado en proyectos(ABP)aparece como una de las estrategias que tienen el propósito de guiar de forma constructiva un desarrollo grupal del conocimiento, a través de una interacción estructurada, orientada y formativa. Teniendo en cuenta la complejidad y los objetivos del método en cuestión, y considerando que el trabajo colaborativo se da cuando existe una reciprocidad entre un conjunto de individuos que saben diferenciar y contrastar sus puntos de vista, de tal manera que llegan a generar un proceso de construcción del conocimiento.

Palabras clave: Aprendizaje Basado en Proyectos, competencias, trabajo colaborativo

\begin{abstract}
Over time, numerous methods of teaching and learning have been proposed and continue to be created and implemented, seeking to move students beyond the memorization of concepts or theories and to analyze and interpret the world in a critical and responsible manner. Project-based learning (PBL) appears as one of the strategies that have the purpose of constructively guiding a group development of knowledge, through a structured, oriented and formative interaction. Taking into account the complexity and objectives of the method in question, and considering that collaborative work occurs when there is a reciprocity between a set of individuals who know how to differentiate and contrast their points of view, in such a way that they generate a process of knowledge construction.
\end{abstract}

Keywords: Project-Based Learning, competencies, collaborative work.

Cómo citar este artículo:

Fajardo Pascagaza, E. \& Gil Bohórquez, B. (2019). El aprendizaje basado en proyectos y su relación con el desarrollo de competencias asociadas al trabajo colaborativo. Revista Amauta, 1/33), pp.103-118. http://dx.doi.org/10.15648/am.33.2019.8

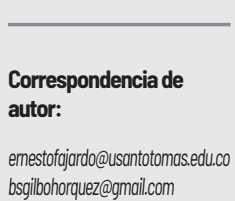

bsgilbohorquez@gmail.com

Recibido:

18/Agosto/2018

Aceptado:

$15 / 0$ ctubre/2018

Publicado:

3/Enero/2018 


\section{Introducción}

Hoy el mundo requiere de nuevas metodologías de educación que fomenten el desarrollo de habilidades para la resolución de problemas, la capacidad de trabajo colaborativo, la toma de decisiones y la adaptación a los repentinos cambios que se producen en la sociedad de la información (Cenich \& Santos, 2005). Por lo tanto, uno de los objetivos principales del proceso de enseñanza-aprendizaje es formar personas capaces de interpretar los fenómenos y sucesos que ocurren a su alrededor (Maldonado, 2008) a través de numerosos métodos que buscan que el estudiante vaya más allá de la memorización de conceptos o teorías y pueda analizar e interpretar el mundo de forma crítica y responsable. Infortunadamente la comunidad educativa con frecuencia asume que dichas innovaciones curriculares son adoptadas por los docentes sin mayor esfuerzo y que tienen efectos rápidos, visibles y directos en las prácticas de enseñanza; por el contrario, Carbonell (2006) indica que los procesos que buscan un cambio educativo suelen ser paulatinos y plantean varios desafíos en las prácticas pedagógicas.

Una de esas metodologías de trabajo es el aprendizaje basado en proyectos (ABP), que según Sanmartí y Márquez (2017):

Parte de aprender alrededor de temáticas complejas que generan interés y tienen sentido para los estudiantes, tiene una larga historia de más de 100 años desde John Dewey, que habló de una enseñanza centrada en el aprendiz 0 activa y William H. Kilpatrick, que la sistematizó en 1918, pasando por Ovide Decroly, Celéstin Freinet y Lawrence Stenhouse, entre otros muchos. También confluyen con estos planteamientos propuestas como las de "Aprendizaje basado en problemas" $y$, más recientemente, en el marco del nuevo currículo de Finlandia, se habla de "Aprendizaje basado en fenómenos". Pero, además se están planteando nuevas propuestas de formatos de aprendizaje como las relacionadas con la "Clase invertida" o las llamadas "Rutinas y estrategias de pensamiento" de David Perkins. Por tanto, nos encontramos ante un amplio abanico de propuestas metodológicas, cada una con objetivos específicos y fundamentaciones teóricas particulares dentro 
de un amplio marco común que tiene como finalidad replantear la actividad educativa. (p.2)

De acuerdo con Lee y Tsai (2004), dicha metodología didáctica es útil especialmente para aquellos cursos o asignaturas de estudios de educación superior que tienen un enfoque general claramente aplicado, ya que promueve el desarrollo de conocimientos con una alta probabilidad de transferirse fuera de los contextos académicos. Pero, el aprendizaje basado en proyectos es una estrategia que tiene como propósito orientar de forma constructiva un desarrollo grupal del conocimiento, a través de una interacción estructurada, orientada y formativa, sin importar el nivel de escolaridad.

Basándonos y tomando principalmente como guía el documento "Aprendizaje por proyectos: una vía al fortalecimiento de los semilleros de investigación" de la autora colombiana Mujica-Rodríguez (2012), el método en cuestión de aprendizaje basado en proyectos emerge de una visión educativa en donde los estudiantes tienen una mayor responsabilidad frente a su propio aprendizaje y aplican en proyectos reales las habilidades y conocimientos adquiridos en el aula. Resulta ser un modelo de aprendizaje en el que el estudiante, no solo crece intelectualmente, sino también como persona, vinculado a una familia, a una sociedad, a un mundo; un estudiante que aprende a aprender, a valorarse, a valorar a los otros y a ver más allá de lo estandarizado.

Si nos referimos al hecho de aprender a valorar a los otros, Guitert y Jiménez (2000) consideran al trabajo colaborativo como una metodología de enseñanza que se da cuando existe una reciprocidad entre un conjunto de individuos que saben diferenciar y contrastar sus puntos de vista, de tal manera que llegan a generar un proceso de construcción del conocimiento, es decir, hacen referencia a un proceso en el que cada persona aprende más de lo que aprendería por sí sola, producto de la interacción de todos los integrantes del grupo de trabajo.

Teniendo en cuenta lo anterior, mediante el presente texto se busca establecer la relación que existe entre el aprendizaje basado en proyectos y el desarrollo de competencias asociadas al trabajo colaborativo. A continuación, se aborda la pregunta en cuestión a través de un desarrollo argumentativo centrado en los planteamientos de 
Mujica-Rodríguez (2012), seguido de autores que aceptan su postura y para finalizar, se establece una serie de conclusiones asociadas al objetivo planteado.

\section{Desarrollo argumentativo}

Ante las necesidades que plantea la actualidad, existen retos en la formación de los estudiantes, requiriendo de metodologías innovadoras y participativas relacionadas con el mejoramiento de la calidad de la enseñanza y el aprendizaje, según los objetivos o metas a las que el docente quiera llegar con el estudiante. Como se mencionó previamente, de acuerdo con la proposición de Mujica-Rodríguez (2012):

Se ha visto que este tipo de aprendizaje basado en proyectos permite al estudiante aprender a investigar, llevando la aplicación de estos conocimientos a otras situaciones; motivarse para que sea él quien resuelva los problemas, plantee y dirija su propio proyecto; se convierta en un descubridor, integrador y presentador de ideas. (p.4)

Así mismo, promueve el uso de la tecnología, el trabajo colaborativo, y, por ende, un desarrollo de habilidades sociales relacionadas con el trabajo en grupo y la negociación (Instituto Tecnológico y de Estudios Superiores de Monterrey, 2006). Además, induce a una participación de todos los actores del aula y propicia su formación integral. Es por esto último que es necesaria la aplicación conjunta del aprendizaje colaborativo, que se funda como un proceso de construcción grupal, a través de la interacción entre los estudiantes, el docente, el contexto y aquello que se quiere comprender.

Todo lo anterior a favor del desarrollo de la habilidad y la capacidad del ser humano de saber interpretar y apreciar los constructos de los demás, para poder asi entender sus propios pensamientos, y aprender para y con los demás (Maldonado, 2007). En este proceso, es fundamental tener en cuenta las opiniones, críticas, comentarios y argumentos de todos los integrantes del grupo, para cumplir logros de forma conjunta, de modo que se favorezcan tanto el crecimiento individual y grupal como sus habilidades, no solo cognitivas, sino también actitudinales y sociales.

De acuerdo con ello, el aprendizaje colaborativo es parte esencial del aprendizaje basado en proyectos, donde se deben organizar grupos de trabajo, a los cuales les 
corresponde aprender a producir en conjunto basados en una pregunta de investigación y/o hipótesis. La organización de los grupos de trabajo, como lo plantea el aprendizaje colaborativo, permitirá una mejor ejecución de las tareas de investigación y búsqueda de la información; logrará que el grupo de trabajo sea más eficiente, proponga y desarrolle su proyecto de manera acertada y según el tiempo estipulado (Mujica-Rodríguez, 2012), además, permitirá a los estudiantes conocerse y aprender a trabajar entre ellos, en busca del cumplimiento de objetivos comunes.

Antes de sustentar la posición de Mujica-Rodríguez (2012), vale la pena señalar que para Vygotsky (1978), la construcción del conocimiento en primera instancia se da gracias a esa interacción social en un contexto de colaboración e intercambio, para luego, individualmente, internalizar los conocimientos adquiridos en grupo y reconstruir los propios. Para Harwell (1997) este modelo de aprendizaje busca desarrollar actividades interdisciplinarias, de mediano a largo plazo, centradas en el estudiante y desde el apoyo del docente como mediador. El planteamiento del aprendizaje basado en proyectos tiene sus orígenes en el constructivismo, que evolucionó a partir de los trabajos realizados por psicólogos y educadores, tales como Lev Vygotsky, Jerome Bruner y John Dewey. Fue este último quien centró su atención en el poder de usar problemas intelectuales y prácticos como vehículo para la enseñanza y el aprendizaje (Dewey, 1929).

Los aportes de Díaz-Barriga (2003) y de De Fillipp (2001), permiten afirmar que el aprendizaje basado en proyectos facilita la integración del conocimiento y su aplicación a situaciones de la realidad. Dicha integración puede darse a partir de la asignación de una actividad con objetivos específicos y su relación con un tópico, problema real o cuestionamientos derivados de alguna experiencia. Las contradicciones, posturas, y debates que surgen, junto a las vías para su solución, contribuyen a que los estudiantes influenciados pedagógicamente se conviertan en sujetos activos.

Por otro lado, en cuanto al rol docente, este modelo de aprendizaje exige que el profesor sea un guía que estimule a los estudiantes a aprender, a descubrir y a sentirse satisfechos por el saber generado, lo cual puede lograrse si aplica correctamente la enseñanza basada en proyectos. En este orden de ideas, para Maldonado (2008) el aprendizaje basado en proyectos: 
Proporciona una experiencia de aprendizaje que involucra al estudiante en una situación compleja y significativa, mediante la cual desarrolla integralmente sus capacidades, habilidades, actitudes y valores. Se acerca a una realidad concreta en un ambiente académico por medio de la realización de un proyecto de trabajo. Estimula en los estudiantes el desarrollo de habilidades para resolver situaciones reales, con lo cual se motivan a aprender; los estudiantes se entusiasman con la investigación, la discusión, proponen y comprueban sus hipótesis, poniendo en práctica sus habilidades en una situación real. En esta experiencia, el estudiante aplica el conocimiento adquirido en un producto dirigido a satisfacer una necesidad social, lo cual refuerza sus valores y su compromiso con el entorno, utilizando además recursos modernos e innovadores. (p. 4)

El aprendizaje basado en proyectos permite integrar la teoría y la práctica, potenciar las habilidades intelectuales superando la mera memorización y repetición, promover valores de responsabilidad personal y de equipo al establecer metas propias; así como fomentar el pensamiento autocrítico y evaluativo. Por consiguiente, el aprendizaje colaborativo se concibe como un acto social en donde deben imperar el diálogo en la construcción del conocimiento y la reflexión para cuestionarse la realidad (García-Valcárcel, 2009). Sustentando lo anterior, cuando se habla de aprendizaje basado en proyectos, también se habla de que estos deben buscar actividades con propósito, por lo tanto, el proyecto debe fundamentarse en los intereses de los alumnos y en los temas del currículo del curso respectivo.

Abordando la pregunta en cuestión, este puede llevarse a cabo en forma individual o colaborativa, siendo la última lo ideal en el propósito de desarrollar habilidades sociales, comunicativas, creativas y en pro del crecimiento del autoestima (Vélez, 1998); y en opinión de Glinz (2005), el trabajo en grupo permite lograr aprendizajes significativos, el desarrollo de habilidades cognitivas como el razonamiento, la observación, el análisis y el juicio crítico, entre otras; al tiempo que se promueve la socialización y la aceptación de las comunidades en las que se trabaja.

Al realizar un énfasis en ¿cómo trabajar con el aprendizaje basado en proyectos? Este es un modelo en el que los estudiantes planean, desarrollan y evalúan proyectos 
que cruzan las fronteras del aula y tienen como objetivo lograr un impacto en la vida real (Challenge 2000 \& Multimedia Project, 2002). La estrategia por proyectos se orienta hacia la elaboración de un proyecto o plan que incluye la realización de tres etapas: planificación, desarrollo y notificación de resultados. En la primera, se identifica el problema y se determinan los recursos necesarios; en la segunda, se investiga sobre la pregunta o tema, se formulan posibles soluciones y se crea un producto, teniendo en cuenta la solución más viable y los resultados de la investigación; y la tercera conlleva la presentación del producto final a los evaluadores correspondientes (Brooks-Young, 2006).

En Mujica-Rodríguez (2012) se distinguen algunas actividades generales y secuenciales asociadas a una propuesta en aprendizaje por proyectos, como la organización de equipos interdisciplinarios, asignación de roles, definición del problema, fundamentación del proyecto, creación de propósitos y objetivos, establecimiento de la metodología, resultados e impacto, prueba piloto, exposición y evaluación.

Respecto a las experiencias de docentes en la aplicación de la enseñanza por proyectos, las investigaciones consultadas dan cuenta de algunos casos. Por ejemplo, Toro (2004) refiere su experiencia al aplicar el aprendizaje basado en proyectos con alumnos de primaria en el área de español; de igual manera, Foulger y Jiménez (2007) afirman que en la enseñanza del inglés el trabajo por proyectos ha demostrado ser efectivo para reforzar el proceso de escritura.

En México, las más recientes reformas curriculares a la educación secundaria incorporaron el aprendizaje basado en proyectos como innovación en ciencias con la intención de mejorar la calidad de la enseñanza y el aprendizaje de estas disciplinas. La incorporación a la propuesta curricular para enseñar ciencias en secundaria no fue algo trivial, por el contrario, planteó varios desafíos a los profesores como abandonar formas de trabajo bastante arraigadas, modificar significativamente sus concepciones y saberes acerca de lo que es la ciencia y lo que implica enseñarla. Además, consiguieron dominar eficazmente y en poco tiempo, una propuesta con la que tenían total falta de familiaridad (Blancas-Hernández \& Guerra-Ramos, 2016).

Para Ronteltap y Eurelings (2002), el objetivo de incluir el trabajo colaborativo en esta propuesta permite crear situaciones en las cuales se generen interacciones productivas entre los alumnos. Esta noción de productividad exige de los estudiantes poner 
en juego estrategias y procesos cognitivos superiores, ya que deben evaluar situaciones contextualizadas, tomar decisiones, proponer soluciones, negociar ideas con base en argumentos referenciados en conocimiento y construir la propuesta (Cenich \& Santos, 2005).

Otro trabajo consistía en indagar las formas en que las estrategias del aprendizaje colaborativo se integran al método de aprendizaje basado en proyectos y se aplican en las aulas, de manera específica en la asignatura de español (Álvarez-Borrego, Herrejón-Otero, Morelos Flores \& Rubio González, 2010). Más relacionado a la pregunta en cuestión, Blanes, Moncada, Casado, Baell \& García (2011) presenta un estudio que consistía en explorar el beneficio que el trabajo colaborativo entre el profesorado puede aportar al diseño y aplicación del aprendizaje basado en proyectos en las nuevas asignaturas de grado en el contexto de la Universidad de Alicante.

Es importante resaltar que existe una serie de metodologías profundamente relacionadas con el aprendizaje por proyectos, que según Badia y García (2006) consiste en la activación, promoción y valoración de los procesos cognitivos, en donde los problemas y tareas se diseñan creativamente. Algunas de ellas son la Situación Problema asociada a procesos de aprendizaje y de construcción de conocimiento, al mundo exterior, la cotidianidad y el contexto (Salas, 2005) o el Aprendizaje Basado en Proyectos Colaborativos (ABPC) que es una metodología didáctica que organiza el proceso de enseñanza y aprendizaje mediante la elaboración de proyectos de forma colaborativa en grupos de estudiantes (La Cueva, 1998).

Por lo general, todos los anteriores tienen una finalidad similar y cuando se habla de un aprendizaje basado en o por proyectos, actualmente se refieren a metodologías muy diversas. Aunque todas tienen algunos rasgos comunes, parten del estudio de alguna situación o problema contextualizado, investigan para dar respuesta a preguntas, dudas o retos, iniciales o que van surgiendo a lo largo de la realización del proyecto; dan a los alumnos la oportunidad de trabajar autónomamente por periodos de tiempo extensos, el profesor facilita pero no dirige, se trabaja en grupos heterogéneos y se promueve la reflexión así como emplear herramientas para aprender de manera interactiva el uso de tecnologías digitales. Por todo ello, esta metodología de trabajo permite fomentar el trabajo colaborativo, además, de utilizar el entorno virtual para la realización conjunta de un proyecto. 
En cuanto a la evaluación, según Sanmartí y Márquez (2017), los estudiantes son evaluados principalmente en función del proyecto realizado y no mediante exámenes u otros instrumentos de evaluación más tradicional y finalizan con alguna acción en el entorno que planifican los propios estudiantes.

Teniendo en cuenta que el ABP es una forma sofisticada y compleja de instrucción, los profesores necesitan del apoyo de sus colegas. Adicionalmente, el trabajo colaborativo entre docentes, en tanto que es una modalidad de aprendizaje entre iguales, constituye una oportunidad de formación y perfeccionamiento con independencia de cuál sea el método instructivo utilizado en el aula (Durán \& Miquel, 2003). En este sentido, los docentes aprenden a partir de la interacción con otros colegas y la colaboración entre ellos se convierte en una herramienta imprescindible para favorecer una práctica reflexiva, así como un recurso esencial para alcanzar la máxima eficacia en su labor docente. Por lo tanto, de la misma manera que se ha hablado del trabajo colaborativo para los estudiantes como una estrategia esencial de aprendizaje, entre los docentes también es un recurso necesario para optimizar el ejercicio de enseñanza.

Emplear el aprendizaje por proyectos como estrategia didáctica es relevante en la experiencia educativa, al considerar que permite la integración de asignaturas, reforzando la visión de conjunto de los saberes humanos, fomentando la responsabilidad individual, el trabajo colaborativo y la capacidad crítica (Maldonado, 2008). Ofrece oportunidades de colaboración para construir conocimiento, aumenta las habilidades comunicativas, permite a los estudiantes compartir ideas entre ellos 0 amplificar las ideas de otros, expresar sus propias opiniones y negociar soluciones.

Trabajar colaborativamente implica compartir experiencias y conocimientos y tener una clara meta grupal. Por ende, lo que debe ser aprendido solo se puede lograr si el trabajo del grupo es realizado en colaboración, considerando que es el grupo el que decide cómo realizar las actividades, los procedimientos a emplear, la distribución del trabajo y las responsabilidades.

Esta metodología didáctica, soportada por interacciones dialógicas, permite enriquecer las producciones, dado que compone los aportes individuales en una sintesis de ideas de todos los miembros del grupo. De esta manera, se observa que 
el trabajo colaborativo consta, como han definido Ingram y Hathorn (2004), de tres elementos decisivos: participación, interacción y síntesis.

Como se ha visto a través del texto, existen otros fines u objetivos en el aprendizaje basado en proyectos, además de los relacionados con el tema objeto de estudio. Procura una mejoría y el desarrollo de competencias en los estudiantes, tales como trabajar en equipo y resolver problemas o tareas complejas, de modo que sean vía para un acercamiento a la investigación. Igualmente, esta estrategia permite mejorar los conocimientos y destrezas frente a las TIC, así como promover la responsabilidad por el propio aprendizaje, esto es, tener autonomía en la adquisición de su saber.

De lo antes mencionado, se pueden destacar dos características centrales y estrechamente relacionadas en el proceso de aprendizaje: la resolución de problemas y el trabajo colaborativo. Para la primera, se requieren buenos problemas, significativos y verdaderamente complejos, que estimulen la exploración y reflexión necesarias para la construcción del conocimiento. La segunda característica se refiere a la interacción con otros, trabajar juntos como pares y aplicar sus conocimientos combinados a la solución del problema. También, se registra una asociación con el planteamiento de Vygotsky (1978), quien denomina la Zona de Desarrollo Próximo (ZDP)como la distancia entre el nivel real de desarrollo, determinado por la capacidad de resolver independientemente un problema, y el nivel de desarrollo potencial, determinado a través de la resolución de un problema bajo la guía de un adulto 0 en colaboración con otro compañero más capaz, nivel que puede ser alcanzado a través del aprendizaje basado en problemas.

En el trabajo colaborativo, los grupos no constituyen el ente fundamental por el simple hecho de colocar personas sentadas en un mismo salón o juntos en un lugar específico, e informarles que son un grupo que debe colaborar. Lo más importante en la formación de grupos de trabajo colaborativo, es supervisar que los elementos básicos estén claramente estructurados en cada sesión de trabajo. Solo de esta manera, se puede lograr que se produzca tanto el esfuerzo colaborativo en el grupo, como una estrecha relación entre la colaboración, el proceso y los resultados. Es importante que el docente influya y dirija sus acciones hacia la interactividad, coordinación, sincronía y el reconocimiento del par en la negociación grupal. 
Las principales ventajas derivadas del uso de estrategias de trabajo colaborativo derivan en el desarrollo y mejora continua de las competencias del docente para ejercer el apoyo y acompañamiento responsables y creativos (Vigotsky, 1978), y en cuanto al estudiante, el desarrollo de competencias sociales, de metacognición y metaevaluación, lo que le confiere mayor autonomía y pertinencia a sus participaciones (Johnson, 1993).

\section{Conclusiones}

Considerando que el aprendizaje basado en proyectos implica formar equipos integrados por personas con perfiles diferentes, áreas disciplinares, profesiones, idiomas y culturas que trabajan juntos para realizar proyectos y solucionar problemas reales, estas diferencias determinan una relación entre los dos grandes conceptos que se han cuestionado directamente, ya que ofrecen grandes oportunidades para el aprendizaje y prepararán a los estudiantes para trabajar en un ambiente y en una economía diversa y global. Además, permite identificar en el aula, mediante la organización y el trabajo por grupos, lo único, diferente y diverso que es cada ser humano, donde cada uno, de acuerdo con su propia meta u objetivo común, en pro de resolver una tarea o un problema, debe trabajar de forma colaborativa durante todo su desarrollo (Corredor, Pérez \& Arbeláez, 2009). Por lo tanto, permite el aprender en la diversidad al trabajar todos juntos, aprender el uno del otro y también la forma de ayudar a que sus compañeros comprendan; los estudiantes aprenden a evaluar el trabajo de sus pares y a dar retroalimentación constructiva tanto para ellos como para sus compañeros.

El trabajo colaborativo, en un contexto educativo como el del objeto de estudio de este escrito, constituye un modelo de aprendizaje interactivo, que invita a los estudiantes a construir juntos, lo cual demanda conjugar esfuerzos, talentos y competencias, mediante una serie de transacciones que les permitan lograr las metas establecidas consensuadamente (Maldonado, 2007). Por lo tanto, es importante perseguir el logro de objetivos a través de la realización (individual y conjunta) de actividades y ser conscientes de que el trabajo colaborativo exige de los participantes habilidades comunicativas, relaciones recíprocas, así como un deseo de compartir la resolución de problemas. 
Son muchas las ventajas que este modelo ofrece al proceso de aprendizaje, ya que promueve que los estudiantes piensen y actúen con base al diseño de un proyecto, elaborando un plan con estrategias definidas, para dar una solución a un interrogante y no tan solo cumplir al pie de la letra los objetivos y contenidos curriculares. Pero, el aprendizaje basado en proyectos es complicado y requiere perseverancia, dedicación y el mejor de los esfuerzos por parte de todos los actores implicados. Sin embargo, el proponer, definir roles y crear modelos innovadores de aprendizaje que logren potenciar las capacidades de autoaprendizaje y desarrollar competencias en nuestros estudiantes es justificable en todos los sentidos.

\section{Referencias bibliográficas.}

Álvarez Borrego, V., Herrejón Otero, V.C., Morelos Flores, M. \& Rubio González, M. T. (2010). Trabajo por proyectos: aprendizaje con sentido. Revista Iberoamericana de Educación, 52(5), 1-13.

Badia, A. \& García, C. (2006). Incorporación de las TIC en la enseñanza y el aprendizaje basados en la elaboración colaborativa de proyectos. Revista de Universidad y Sociedad del Conocimiento (RUSC), 3(2), 1-13.

Blancas Hernández, J. L. \& Guerra Ramos, M. T. (2016). Trabajo por proyectos en el aula de ciencias de secundaria: tensiones curriculares y resoluciones docentes. Revista Mexicana de Investigación Educativa, 21(68), 141-166.

Blanes, M. D., Moncada, R. O., Casado, D. L. P., Baell, I. M. \& García, J. Á. (2011). Trabajo colaborativo para la aplicación del aprendizaje basado en proyectos en asignaturas de grado. IX Jornadas de Redes de Investigación en Docencia Universitaria: Diseño de buenas prácticas docentes en el contexto actual (p.108). Universitat d'Alacant.

Brooks Young, S. (2006). Critical Technology Issues for School Leaders. Thousand Oaks, California: Corwin Press.

Carbonell, J. (2006). La aventura de innovar. El cambio en la escuela. Madrid: Morata.

Cenich, G. \& Santos G. (2005). Propuesta de aprendizaje basado en proyectos y trabajo colaborativo: experiencia de un curso en línea. Revista Electrónica de Investigación Educativa, 72). Consultado el 18 de septiembre de 2018 en: http:// redie.uabc.mx/vol7no2/contenido-cenich.html 
Challenge 2000 \& Multimedia Project (2002). Why Do Project Based Learning? San Mateo, California: San Mateo County Office of Education. Consultado el 18 de septiembre de 2018 en: http://pblmm.k12.ca.us/PBLGuide/WhyPBL.html

Corredor, M. V., Pérez, M. I. \& Arbeláez, R. (2009). Estrategias de enseñanza y aprendizaje. Bucaramanga: Publicaciones UIS.

De Fillippi, R. J. (2001). Introduction: Project-based learning, reflective practices and learning outcomes. Management Learning, 32(1), 5-11.

Dewey, J. (1929). The Quest for Certainty: A Study of the Relation of Knowledge and Action. Nueva York: Minton, Balch \& Co.

Díaz Barriga, A. F. (2003). Cognición situada y estrategias para el aprendizaje significativo. Revista Electrónica de Investigación Educativa, 5(2), 1-13.

Durán, D. \& Miquel, E. (2003). Cooperar para enseñar y aprender. Cuadernos de Pedagogía, 331, 73-76.

Estrada García, A. (2012). El aprendizaje por proyectos y el trabajo colaborativo, como herramientas de aprendizaje, en la construcción del proceso educativo, de la Unidad de aprendizaje TIC. Revista Iberoamericana para la Investigación y el Desarrollo Educativo, 3(5), 123-138.

Foulger, T. \& Jimenez, M. (2007). Enhancing the Writing Development of English Language Learners: Teacher Perceptions of Common Technology in ProjectBased Learning. Journal of Research in Childhood Education, 22(2), 109-124.

García-Valcárcel, A. (2009). Modelos y estrategias de enseñanza. Videoconferencia presentada como parte de los documentos de la materia Modelos y Estrategias de Enseñanza, de la Maestría en Educación del Instituto Tecnológico de Monterrey.

Garmendia, M., Fuentes, J. I. B., Elosegi, K. Z. \& Aranzábal, J. G. (2014). Proyecto de formación del profesorado universitario de Ciencias, Matemáticas y Tecnología, en las metodologías de Aprendizaje Basado en Problemas y Proyectos. Enseñanza de las ciencias, 32(2), 113-129.

Glinz Férrez, P. E. (2005). Un acercamiento al trabajo colaborativo. Revista Iberoamericana de Educación, 35(2). Consultado el 18 de septiembre de 2018 en: http:// www.rieoei.org/deloslectores/820Glinz.PDF

Guitert, M. \& Jiménez, F. (2000). Trabajo cooperativo en entornos virtuales de aprendizaje. En: Aprender de la Virtualidad. España: Edit. Gedisa.

Johnson, D. W., Johnson, R. T. \& Smith, K. A. (1991). Cooperative Learning: Increasing College Faculty Instruction Productivity. ASHE-ERIC Higher Education Report No. 
4. Washington, DC: The George Washington University, School of Education and Human Development.

Harwell, S. (1997). Project-based learning. In W.E. Blank \& S. Harwell (eds.), Promising Practices for Connecting High School to the Real World. Tampa: University of South Florida.

Ingram, A. \& Hathorn L. (2004). Methods for analyzing collaboration in online communications. In T. Roberts (Ed.), Online collaborative learning: Theory and practice (pp. 215-241). Hershey, PA: Idea Group Publishing.

Instituto Tecnológico y de Estudios Superiores de Monterrey (ITESM). (2006). Las estrategias y técnicas didácticas en el rediseño: El método de proyectos como técnica didáctica. Monterrey: Instituto Tecnológico y de Estudios Superiores de Monterrey. Consultado el 18 de septiembre de 2018 en: http://www.sistema. itesm.mx/va/dide/inf-doc/estrategias/

La Cueva, A. (1998). La enseñanza por proyectos: ¿mito o reto? Revista Iberoamericana de Educación, 16, 165-187.

Lee, C. I. \& Tsai, F. Y. (2004). Internet project-based learning environment: the effects on thinking styles on learning transfer. Journal of Computer Assisted Learning, 20, 1-39.

Maldonado, M. (2007). Aprendizaje Basado en Proyectos en la Educación Técnica. Ponencia presentada en el Congreso Internacional de Educación Técnica, Universidad Pedagógica Experimental Libertador. Barquisimeto, Venezuela.

Maldonado, M. (2008). Aprendizaje basado en proyectos colaborativos. Una experiencia en educación superior. Laurus, 14(28), 158-180.

Mujica Rodríguez, A. M. (2012). Aprendizaje por proyectos: Una vía al fortalecimiento de los semilleros de investigación. Docencia Universitaria, 13, 201-216.

Revelo Sánchez, 0., Collazos-Ordóñez, C. A., \& Jiménez-Toledo, J. A. (2018). El trabajo colaborativo como estrategia didáctica para la enseñanza/aprendizaje de la programación: una revisión sistemática de literatura. TecnoLógicas, 21 (41), 115-134.

Ronteltap, F. \& Eurelings, A. (2002). Activity and interaction of students in an electronic learning environment for problem-based learning. Distance Education, $23(1), 11-22$.

Salas, W. A. (2005). Formación por competencias en educación superior. Una aproximación conceptual a propósito del caso colombiano. Revista Iberoamericana de 
Educación, 36(9). Consultado el 18 de septiembre de 2018 en: http://www.rieoei. org/deloslectores/1036Salas.PDF

Sanmartí, N. \& Márquez, C. (2017). Aprendizaje de las ciencias basado en proyectos: del contexto a la acción. Ápice. Revista de Educación Científica, 1(1), 3-16.

Toro, L. (2004). Los docentes como diseñadores de enseñanza. Revista Iberoamericana de Educación, 34(4). Consultado el 18 de septiembre de 2018 en: http:// www.rieoei.org/deloslectores/802Toro.PDF

Vélez, A. (1998). Aprendizaje basado en proyectos colaborativos en la educación superior. IV Congresso RIBIE. Brasilia.

Vygotsky, L. S. (1978). Desarrollo de los procesos psicológicos superiores. Primera edición. Barcelona: Grijalbo. 